\title{
Non-Gaussian behavior of crystalline and amorphous phases of polyethylene
}

\author{
T. Kanaya* \\ Institute for Chemical Research, Kyoto University, Uji, Kyoto-fu 611 0011, Japan \\ U. Buchenau and S. Koizumi \\ Institut für Festkörperforschung, Forschungszentrum, D-52425 Jülich, Germany \\ I. Tsukushi and K. Kaji \\ Institute for Chemical Research, Kyoto University, Uji, Kyoto-fu 611 0011, Japan
}

(Received 8 June 1999)

\begin{abstract}
We report on measurements of the incoherent elastic neutron-scattering intensity $I_{\mathrm{el}}(Q)$ of polyethylenes with degrees of crystallinity 0.46 and 0.96 in a wide $Q$ (length of scattering vector) range from 0.4 to $6.2 \AA^{-1}$ to observe the deviation from the Gaussian behavior. The non-Gaussian behavior was observed in both the amorphous and the crystalline phases. The non-Gaussian behavior in the theoretically well-known crystalline phase can be understood more or less quantitatively in terms of the anisotropy of the mean-square displacement. The result implies that at least a part of the non-Gaussian behavior of the amorphous phase is due to the same source, while another part may be due to the dynamical heterogeneity inherent to the amorphous phase.
\end{abstract}

In the incoherent elastic scattering from solids, one often finds deviations from the Gaussian behavior ( $2 \mathrm{~W}$ $=\alpha Q^{2} ; 2 W$ and $\alpha$ are exponents of the Debye-Waller factor and mean-square displacement of the atoms in the direction of the momentum transfer $Q$ ). These deviations are denoted as non-Gaussian behavior. In amorphous solids, where the low-frequency excitations are only poorly understood, ${ }^{1,2}$ one has evidence for localized low-frequency modes from numerical work, ${ }^{3-5}$ so it seems natural to attribute the nonGaussianity to these localized modes, ${ }^{6}$ in the spirit of the soft potential model ${ }^{7,8}$ which postulates a continuous crossover from the low-barrier tunneling modes to the additional vibrational modes. The non-Gaussianity of amorphous polymers has been seen even more clearly in elastic measurements with good resolution ${ }^{9-11}$ up to a rather high $Q$.

In this work, incoherent elastic neutron-scattering measurements have been performed on amorphous and crystalline phases of polyethylene in a wide $Q$ range to see the non-Gaussian behavior. One finds a strong non-Gaussianity also for the crystalline case, where one has no low-frequency localized modes. It turns out that it is possible to understand this non-Gaussianity in terms of the hydrogen vibrations in the crystalline structure. On the basis of the results, we will discuss the origin of the non-Gaussian behavior for both the crystalline and amorphous phases.

Two types of polyethylenes (PE) were used in this experiment: semicrystalline and highly crystalline PE's with degrees of crystallinity 0.46 and 0.96 , respectively. The former was a low-density PE (Sumikasene G806, Sumitomo Chemical Industrial Co., Ltd.) with a molecular weight of 80600. The latter was prepared by fractionating high-density PE (Sholex 6009, Japan Olefin Chem. Ltd.) and crystallizing at $129^{\circ} \mathrm{C}$ for 15 days. The molecular weight was 11000 . The degrees of crystallinities were determined by density measurements and broadline NMR spectroscopy. It should be emphasized that the crystallites in our samples were not mac- roscopically oriented; one measures a polycrystalline average. Thus, the difference in mean-square displacement along the chain $\alpha_{\|}$and perpendicular to the chain $\alpha_{\perp}$ in the crystal contributes to the measured non-Gaussian behavior.

Incoherent elastic-scattering measurements were performed with a triple axis spectrometer (GP-TAS) installed at a thermal neutron source in the JRR-3M reactor, Tokai. The incident energy of neutrons was $30 \mathrm{meV}$ and the energy resolution evaluated from the full width at half maximum (FWHM) was $1.6 \mathrm{meV}$. Under this condition, the spectrometer can cover a $Q$ range from 0.4 to $6.4 \AA^{-1}$.

After subtracting the empty can scattering, the observed incoherent elastic neutron-scattering intensities have been corrected for multiple scattering, ${ }^{12}$ which affects the results seriously, especially at low temperatures below $\sim 100 \mathrm{~K}$. The observed elastic-scattering intensity $I_{\mathrm{el}, p}(Q)$ of PE with degree of crystallinity $p$ was assumed to be a linear combination of those of the crystalline phase $I_{\mathrm{el}, c}(Q)$ and the amorphous phase $I_{\mathrm{el}, a}(Q)$ weighted by $p$ and $1-p$, respectively,

$$
I_{\mathrm{el}, p}(Q)=p I_{\mathrm{el}, c}(Q)+(1-p) I_{\mathrm{el}, a}(Q) .
$$

Using the data for $p=0.46$ and 0.96 , we have calculated the elastic intensities of the crystalline phase $I_{\mathrm{el}, c}(Q)$ and the amorphous phase $I_{\mathrm{el}, a}(Q)$ and the logarithms are plotted versus $Q^{2}$ in Fig. 1, where $I_{\mathrm{el}, c}(Q)$ and $I_{\mathrm{el}, a}(Q)$ are divided by those at $10 \mathrm{~K}$ to reduce the effects of the coherent scattering.

Assuming a Gaussian distribution of the mean-square displacement $\alpha$ (Gaussian approximation), the $Q$ dependence of incoherent elastic-scattering intensity can be described by

$$
I_{\mathrm{el}}(Q)=\exp \left(-\alpha Q^{2}\right) \text {. }
$$

This relation is sustained in a low- $Q$ range in any system whether it is amorphous or crystalline. ${ }^{13}$ It is clear from Fig. 1 that $I_{\mathrm{el}}(Q)$ 's for the crystalline and amorphous phases deviate from the relation of Eq. (2), showing the non-Gaussian 


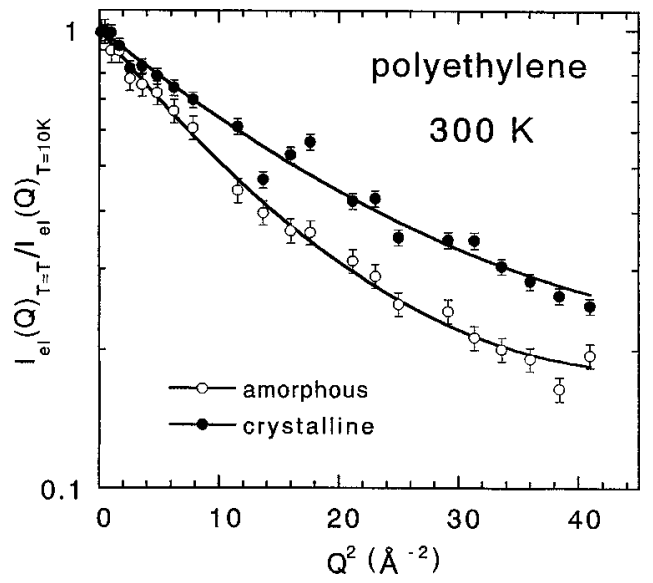

FIG. 1. Logarithm of incoherent elastic neutron-scattering intensity $I_{\mathrm{el}}(Q)$ for crystalline and amorphous phases of polyethylene as a function of $Q^{2}$ at $300 \mathrm{~K}$. Elastic intensity $I_{\mathrm{el}}(Q)_{T=T}$ was divided by $I_{\mathrm{el}}(Q)_{T=10 \mathrm{~K}}$ at $10 \mathrm{~K}$. The solids curves are the results of fits with Eq. (3) (see text).

behavior in the high- $Q$ range. It was recently shown ${ }^{9}$ that the non-Gaussian behavior arises from many reasons such as dynamic heterogeneity, microscopic anisotropy, and anharmonicity. The $Q$ dependence of $I_{\mathrm{el}}(Q)$ can be approximated up to the order of $Q^{4}$ in the following form: ${ }^{6,10,11}$

$$
I_{\mathrm{el}}(Q)=\exp \left(-\bar{\alpha} Q^{2}+\frac{A_{0} \bar{\alpha}^{2}}{2} Q^{4}\right),
$$

where $\bar{\alpha}$ and $A_{0}$ are an average of the mean-square displacements and a non-Gaussian parameter. Equation (3) was fitted to the observed elastic-scattering intensity to evaluate the average of the mean-square displacements $\bar{\alpha}$ and the nonGaussian parameter $A_{0}$. The results of the fits are shown in Fig. 1 as solid curves, showing a good fit. It should be emphasized that the average of the mean-square displacements $\bar{\alpha}$ evaluated here does not include the contributions of $\bar{\alpha}$ at $10 \mathrm{~K}$ because the observed elastic-scattering intensity is divided by that at $10 \mathrm{~K}$ in Fig. 1 . The average of the real mean-square displacements is given by

$$
\bar{\alpha}=\bar{\alpha}_{\mathrm{obs}}+\bar{\alpha}_{10 \mathrm{~K}},
$$

where $\bar{\alpha}_{10 \mathrm{~K}}$ is the average of the mean-square displacements at $10 \mathrm{~K}$. In this work, we have evaluated the $\bar{\alpha}_{10 \mathrm{~K}}$ from the density of vibrational states ${ }^{14}$ for the low-frequency modes below $\sim 15 \mathrm{meV}$ and from the normal-mode calculation ${ }^{15}$ for the high-frequency modes such as bending and stretching modes. The contribution of the former to $\bar{\alpha}_{10 \mathrm{~K}}$ is 0.0042 and $0.0108 \AA^{2}$ for the crystalline and amorphous phases, respectively, and that of the latter is $0.011 \AA^{2}$ for both the phases. Then, the observed average of the mean-square displacement $\bar{\alpha}_{\text {obs }}$ and the non-Gaussian parameter $A_{0}$ were corrected for the effects of $\bar{\alpha}_{10 \mathrm{~K}}$. The corrected $\bar{\alpha}$ and $A_{0}$ are plotted in Figs. 2(a) and (b), respectively, for the crystalline and amorphous phases as a function of temperature. The average of the mean-square displacement in the amorphous phase $\bar{\alpha}_{a}$ is larger than that of the crystalline phase $\bar{\alpha}_{c}$. This result is very natural because motions of hydrogen atoms in the crystalline phase are suppressed much more than in the amorphous phase. On the other hand, it is very interesting that the

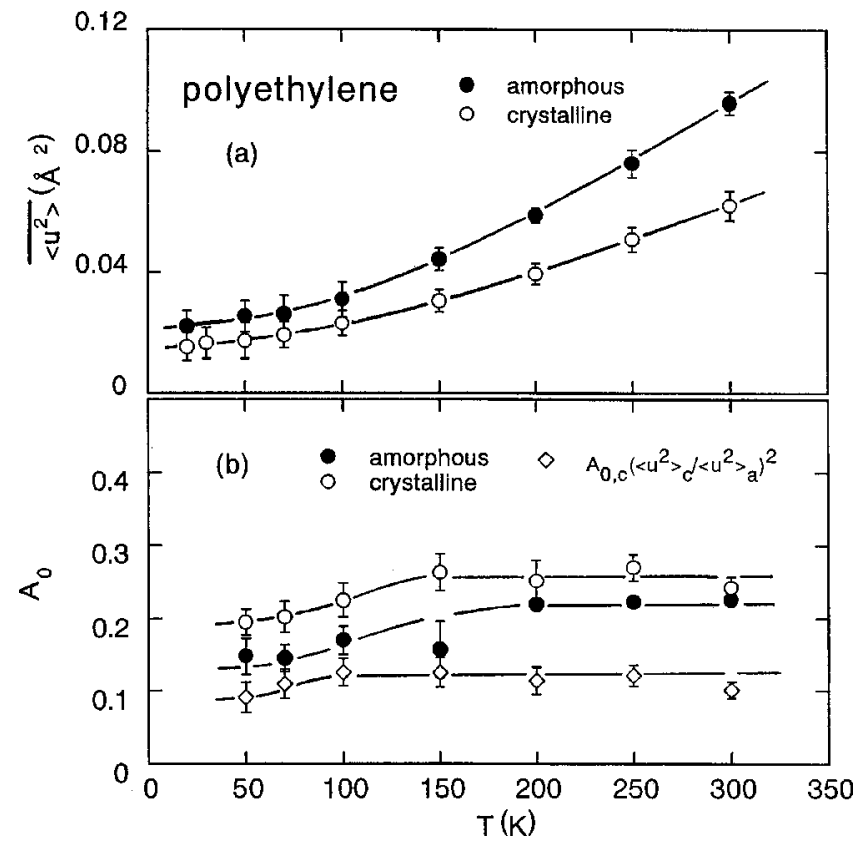

FIG. 2. (a) Average of mean-square displacement $\overline{\left\langle u^{2}\right\rangle}$, (b) nonGaussian parameter $A_{0}$ for crystalline and amorphous phases of polyethylene as a function of temperature. The lines were drawn by eye.

non-Gaussian parameter $A_{0}$ is larger in the crystalline phase than in the amorphous phase. In previous studies on amorphous polymers, ${ }^{10,11}$ the non-Gaussian parameter has been interpreted as a measure of dynamic heterogeneity due to different local environments around an individual molecule. On the other hand, it is obvious that the crystalline phase is more homogeneous than the amorphous phase, at least on a molecular level although it is anisotropic; the displacement of hydrogen atom is smaller in the direction parallel to the chain $\overline{\alpha_{\|}}$than in the direction perpendicular to the chain $\overline{\alpha_{\perp}}$. This anisotropy must contribute to the non-Gaussian parameter in the crystalline phase. In the case of $\overline{\alpha_{\|}}=0.5$ and $\overline{\alpha_{\perp}}$ $=1$, for example, the non-Gaussian parameter $A_{0}$ is calculated to be 0.08 . In the amorphous phase, where neighboring polymer chains still tend to lie parallel to each other, but with a considerable amount of disorder, the difference between $\overline{\alpha_{\|}}$and $\overline{\alpha_{\perp}}$ will still persist, but could be smaller than in the well-ordered crystalline phase.

The mean-square displacement of the amorphous phase has a larger value than that of the crystalline phase, as seen in Fig. 2(a). Representing the excess value by $\Delta \alpha$, the meansquare displacement in the amorphous phase can be described by

$$
\overline{\alpha_{a}}=\overline{\alpha_{c}}+\Delta \alpha
$$

If we assume that $\Delta \alpha$ is the same for all hydrogen atoms in the amorphous phase, termed homogeneous assumption, and the anisotropy of the mean-square displacement in the amorphous phase is the same as that in the crystalline phase, the non-Gaussian parameter $A_{0, a}$ of the amorphous phase is given by

$$
A_{0, a}=A_{0, c}\left(\frac{\overline{\alpha_{c}}}{\overline{\alpha_{a}}}\right)^{2}
$$


Using the observed $\overline{\alpha_{c}}, \overline{\alpha_{a}}$, and $A_{0, c}$ we calculated the nonGaussian parameter $A_{0, a}$ of the amorphous phase through Eq. (6) and plotted as diamonds in Fig. 2. The calculated $A_{0, a}$ 's $\left[=A_{0, c}\left(\overline{\alpha_{c}} / \overline{\alpha_{a}}\right)^{2}\right]$ are almost independent of temperature and at around 0.1 , which is rather smaller than the observed $A_{0, a}$. Note that the calculated $A_{0, c}\left(\overline{\alpha_{c}} / \overline{\alpha_{a}}\right)^{2}$ will probably overestimate the non-Gaussianity from the chain anisotropy in the amorphous phase because this anisotropy will tend to be less marked in the presence of a strong disorder. Hence, the fact $A_{0, a}>A_{0, c}\left(\overline{\alpha_{c}} / \overline{\alpha_{a}}\right)^{2}$ suggests that the homogeneous assumption is not valid for the excess mean-square displacement $\Delta \alpha$ in the amorphous phase, i.e., the additional mean-square displacement $\Delta \alpha$ must have a distribution (heterogeneous assumption) which may be mainly caused by heterogeneous environments around individual atoms. Under this heterogeneous assumption, the non-Gaussian parameter $A_{0, a}$ of the amorphous phase is described as

$$
A_{0, a}=A_{0, c}\left(\frac{\overline{\alpha_{c}}}{\overline{\alpha_{a}}}\right)^{2}+\frac{\overline{\Delta \alpha^{2}}-\overline{\Delta \alpha^{2}}}{\overline{\Delta \alpha^{2}}}\left(\frac{\overline{\Delta \alpha}}{\overline{\alpha_{a}}}\right)^{2} .
$$

The heterogeneous distribution of the excess mean-square displacement produces an additional contribution to the nonGaussian parameter $\left\{\left(\overline{\Delta \alpha^{2}}-\overline{\Delta \alpha}^{2}\right) / \overline{\Delta \alpha^{2}}\right\}\left(\overline{\Delta \alpha} / \overline{\alpha_{a}}\right)^{2}$. The difference between the observed $A_{0, a}$ and the calculated $A_{0, c}\left(\overline{\alpha_{c}} / \overline{\alpha_{a}}\right)^{2}$ may be attributed to the additional contribution originated from the heterogeneous distribution of the excess mean-square displacement. As seen in Fig. 2(b), the difference between $A_{0, a}$ and $A_{0, c}\left(\overline{\alpha_{c}} / \overline{\alpha_{a}}\right)^{2}$ is ranging from 0.05 to 0.1 , corresponding to the contribution of the heterogeneity of the amorphous phase. Note that the value of 0.05 to 0.1 is the lowest because the anisotropy in the amorphous phase must be smaller than in the crystalline phase. This heterogeneity may be an indication of vibrational localization of lowfrequency modes in the amorphous phase. ${ }^{6}$ In the following we will compare our results for the crystalline phase with literature data, experimental as well as theoretical ones, in order to confirm whether or not the present results are reasonable.

Temperature factors $B$ of carbon and deuterium atoms were estimated for the $a, b$, and $c$ directions in the crystalline phase of deuterated polyethylene (d-PE) at 5, 80, and $300 \mathrm{~K}$ by neutron-diffraction measurements. ${ }^{16}$ On the basis of the data, we calculated the average of the mean-square displacements $\overline{\alpha_{c}}$ and the non-Gaussian parameter $A_{0, c}$ of the crystalline phase. Correcting the mass effect, the average of the mean-square displacements calculated from the temperature factor $B \quad\left(=8 \pi^{2} \alpha / 3\right)$ shows a good agreement with the present data while the calculated non-Gaussian parameter $A_{0, c}$ is larger than the present result, especially at 5 and $80 \mathrm{~K}$ as shown in Fig. 3. This disagreement is due to a large anisotropy of the temperature factor at 5 and $80 \mathrm{~K}$. Neutronscattering measurements were also performed by Lynch et al. ${ }^{17}$ and Myers and Randolph ${ }^{18}$ on highly stretch-oriented PE to obtain the Debye-Waller factor parallel and perpendicular to the oriented direction. The averages of the meansquare displacements evaluated from these experiments agree with the observation in this work. However, the nonGaussian parameters $A_{0}$ evaluated from the anisotropy of the macroscopically oriented sample are much smaller than the

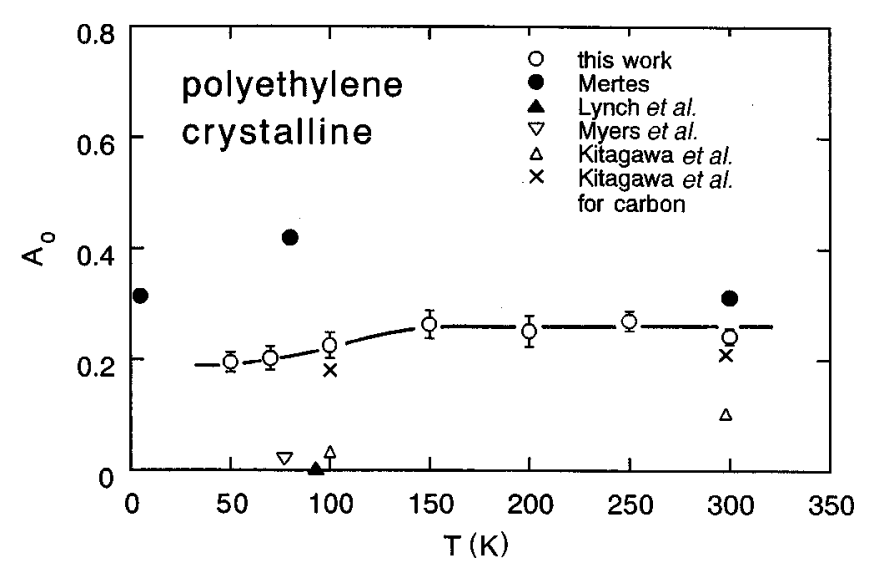

FIG. 3. Comparison of non-Gaussian parameter $A_{0}$ in crystalline phase of polyethylene; $(\bigcirc)$ : this work, $(\bigcirc)$ : neutron diffraction of d-PE, Mertes (Ref. 16), ( $\triangle$ ): incoherent elastic neutron scattering of uniaxially oriented PE, Lynch et al. (Ref. 17), ( $\mathbf{\Delta})$ : incoherent elastic neutron scattering of uniaxially oriented PE, Myers and Randolph (Ref. 18), $(\nabla)$ : calculation on uniaxially oriented PE, Kitagawa et al. (Ref. 19), (x): calculation of temperature factor of carbon in crystalline phase, Kitagawa et al. (Ref. 19). The line was drawn by eye.

present value (see Fig. 3). These small values must be because the crystallinity of the used sample was not high (80$90 \%$ ) and the uniaxial orientation of chain axis was not perfect. The Debye-Waller factors of uniaxially oriented PE were calculated by Kitagawa and Miyazawa ${ }^{19}$ for the same condition as the experiment. ${ }^{17}$ The non-Gaussian parameters $A_{0}$ evaluated from the calculated Debye-Waller factors parallel and perpendicular to the oriented direction are plotted in Fig. 3, which are larger than the values of Lynch et al. ${ }^{17}$ and Myers and Randolph ${ }^{18}$ but still smaller than the value of $A_{0, c}$ of this work. Kitagawa and Randolph ${ }^{19}$ also calculated the temperature factor of carbon atoms for the $a, b$, and $c$ directions in the crystalline phase. The non-Gaussian parameter $A_{0}$ evaluated from the calculated temperature factors is also shown in Fig. 3 and is close to the observed value of $A_{0, c}$. Although the values of $A_{0, c}$ in Fig. 3 show a strong scatter, these comparisons show that the non-Gaussian parameter $A_{0}$ in the crystalline phase surely arises from the anisotropy of the mean-square displacements and the values are not far from the present observation.

As for the amorphous phase, there are no experimental data which can be directly compared with the present result although some experimental works have reported the meansquare displacement of PE in the amorphous phase. ${ }^{20,21} \mathrm{Re}-$ cently, Roe performed a molecular-dynamics (MD) simulation on an amorphous polyethylene model ${ }^{22}$ and calculated the mean-square displacement and the non-Gaussian parameter as a function of time. The present measurements of the non-Gaussian parameter were performed in a frequency domain with an energy resolution FWHM of $1.6 \mathrm{meV}$, approximately corresponding to a time of $\sim 0.8 \mathrm{ps}$. Then, the nonGaussian parameters of the MD simulation are plotted in Fig. 4 at $t=0.5$ and 1 ps for comparison with the experimental value. The values of $A_{0, a}$ from the MD simulation are around at $0.15-0.2$. This value is larger than the non-Gaussian parameter due to the anisotropy $A_{0, c}\left(\overline{\alpha_{c}} / \overline{\alpha_{a}}\right)^{2}$, suggesting that the contribution of the dynamic heterogeneity to the non- 


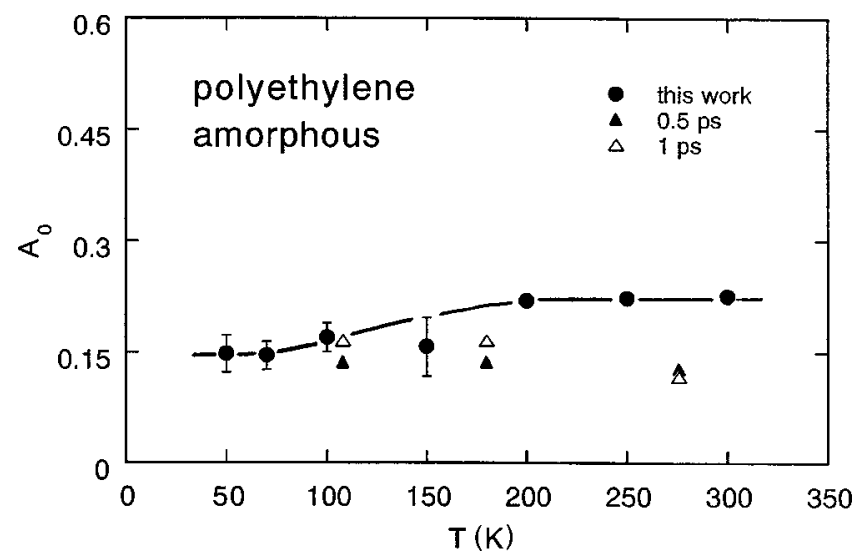

FIG. 4. Comparison of non-Gaussian parameter $A_{0}$ in amorphous phase of polyethylene. $(\mathbf{O})$ : this work, $(\mathbf{\Delta})$ : MD simulation by Roe (Ref. 22) at $t=0.5 \mathrm{ps},(\triangle)$ : at $=1 \mathrm{ps}$. The line was drawn by eye.

Gaussian parameter is not negligible in the amorphous phase, but it is slightly lower than the observed value of $A_{0, a}$. This may suggest that PE in the MD simulation is less heterogeneous than the real system because of the limitation of the cell size. In this study non-Gaussianity due to anharmonicity has been neglected because $\left\langle u^{2}\right\rangle$ is almost proportional to $T$ except for the effect of zero-point vibrations and the inelastic scattering intensity is Bose scaled, at least below $150 \mathrm{~K}$, which suggest the vibrational motions observed here are harmonic. However, the anharmonic effect may not be negligible above the glass transition temperature $T_{g}[\approx 200 \mathrm{~K}$ (Ref. 23)].

In summary, we have shown that the crystalline phase of polyethylene shows a clear non-Gaussian behavior as well as the amorphous phase. It was surprisingly found that the evaluated non-Gaussian parameter $A_{0, c}$ in the crystalline phase is larger than that $A_{0, a}$ in the amorphous phase while the mean-square displacement in the crystalline phase is smaller than in the amorphous phase. The predicted value of $A_{0, a}$ under an assumption of the homogeneous distribution of the additional mean-square displacement $\Delta \alpha$ in the amorphous phase is smaller than the observed one, suggesting that $\Delta \alpha$ has a distribution probably due to the heterogeneous local environments around the individual molecule in the amorphous phase. In other words, the non-Gaussian parameter in the amorphous phase $A_{0, a}$ involves contributions due to the dynamic heterogeneity in addition to the anisotropy of the displacement.

We are grateful to Professor T. Odagaki, Dr. R. Zorn, and Professor K. Tashiro for valuable discussions. This work is partly supported by a grant for Joint Research Project under the Japanese-German Cooperative Science Promotion Program from both Japan Society for the Promotion of Science and Deutsche Forschungsgemeinschaft.
*Author to whom correspondence should be addressed.

${ }^{1}$ Proceedings of Yukawa International Seminar (YKIS96): Dynamics of Glass Transition and Related Topics, edited by T. Odagaki, Y. Hiwatari, and J. Matsui [Prog. Theor. Phys. Suppl. 126 (1997)] and references therein.

${ }^{2}$ Proceedings of The Third International Discussion Meeting on Relaxation in Complex Systems, edited by K. L. Ngai, E. Riande, and M. D. Ingram [J. Non-Cryst. Solids 235/237 (1998)] and references therein.

${ }^{3}$ B. B. Laird and H. R. Schober, Phys. Rev. Lett. 66, 636 (1991).

${ }^{4}$ H. R. Schober and B. B. Laird, Phys. Rev. B 44, 6746 (1991).

${ }^{5}$ H. R. Schober and C. Oligschleger, Phys. Rev. B 53, 11469 (1996).

${ }^{6}$ U. Buchenau, C. Pecharroman, R. Zorn, and B. Frick, Phys. Rev. Lett. 77, 659 (1996).

${ }^{7}$ U. Buchenau, Y. M. Galperin, V. L. Gurevich, and H. R. Schober, Phys. Rev. B 43, 5039 (1991).

${ }^{8}$ I. Tsukushi, T. Kanaya, and K. Kaji, J. Non-Cryst. Solids 235/ 237, 250 (1998).

${ }^{9}$ R. Zorn, Phys. Rev. B 55, 6249 (1997).

${ }^{10}$ T. Kanaya, I. Tsukushi, and K. Kaji, Prog. Theor. Phys. Suppl. 126, 133 (1997).

${ }^{11}$ T. Kanaya, I. Tsukushi, K. Kaji, B. Gabrys, and S. M. Benning- ton, J. Non-Cryst. Solids 235/237, 212 (1998).

${ }^{12}$ T. Kanaya and U. Buchenau (unpublished).

${ }^{13}$ W. Marshall and S. W. Lovesey, Theory of Thermal Neutron Scattering (Clarendon Press, Oxford, 1971), pp. 74 and 375 for crystalline and amorphous systems, respectively.

${ }^{14}$ T. Kanaya, K. Kaji, S. Ikeda, and K. Inoue, Chem. Phys. Lett. 150, 334 (1988).

${ }^{15}$ T. Tasumi and S. Krim, J. Chem. Phys. 46, 755 (1967).

${ }^{16}$ J. Mertes, Ph.D. thesis, Mainz University, 1979.

${ }^{17}$ J. E. Lynch, G. C. Summerfield, L. A. Feldlamp, and J. S. King, J. Chem. Phys. 48, 912 (1968).

${ }^{18}$ W. R. Myers and P. D. Randolph, J. Chem. Phys. 49, 1043 (1968).

${ }^{19}$ T. Kitagawa and T. Miyazawa, Adv. Polym. Sci. 9, 335 (1973).

${ }^{20}$ U. Buchenau, M. Monkenbusch, M. Stamm, C. F. Majkrzak, and N. Nücker, in Polymer Motion in Dense System, edited by D. Richter and T. Springer, Proceedings in Physics (Springer, Berlin, 1988), Vol. 29, p. 138.

${ }^{21}$ G. Hohlweg, B. Holzer, W. Petry, G. Strobl, and B. Stühn, Macromolecules 25, 6248 (1992).

${ }^{22}$ R. J. Roe, J. Chem. Phys. 100, 1610 (1994).

${ }^{23}$ D. W. v. Krevelen, Properties of Polymers (Elsevier, Amsterdam, 1990), p. 125. 